\title{
Hardware and Software Integration to Support Real-Time Space Link Emulation
}

Prior to operational use, communications hardware and software must be thoroughly tested and verified. In space-link communications, field testing equipment can be prohibitively expensive and cannot test to non-ideal situations. In this paper, we show how software and hardware emulation tools can be used to accurately model the characteristics of a satellite communication channel in a lab environment. We describe some of the challenges associated with developing an emulation lab and present results to demonstrate the channel modeling. We then show how network emulation software can be used to extend a hardware emulation model without requiring additional network and channel simulation hardware. 


\section{Overview}

- Introduction

- System Model

- Direction Communication Channel Model

- Bent-pipe Communication Channel Model

- Space-Link Emulation

- Hardware

- Software Modeling

- Results 


\section{Introduction}

- Emulation vs. Simulation

- Emulation must be high-fidelity

- Can interact with field equipment

- Emulation must be performed in real-time

- Benefits of emulation:

- Testing on hardware in the field can be prohibitively expensive

- Testing of 'non-ideal' scenarios is feasible

- Challenges for Emulation:

- Emulation uses real equipment

- Routers, Modems, Switches, etc

- Characteristics of emulation equipment should not impact emulation results

- Have to account for these offsets in the link budget analysis 


\section{System Model}

- Single Channel Emulation

- Direct Comm. Ground Terminal (GT) to Low Earth Orbit (LEO) Satellite

- Two Channels:

- Forward and Return
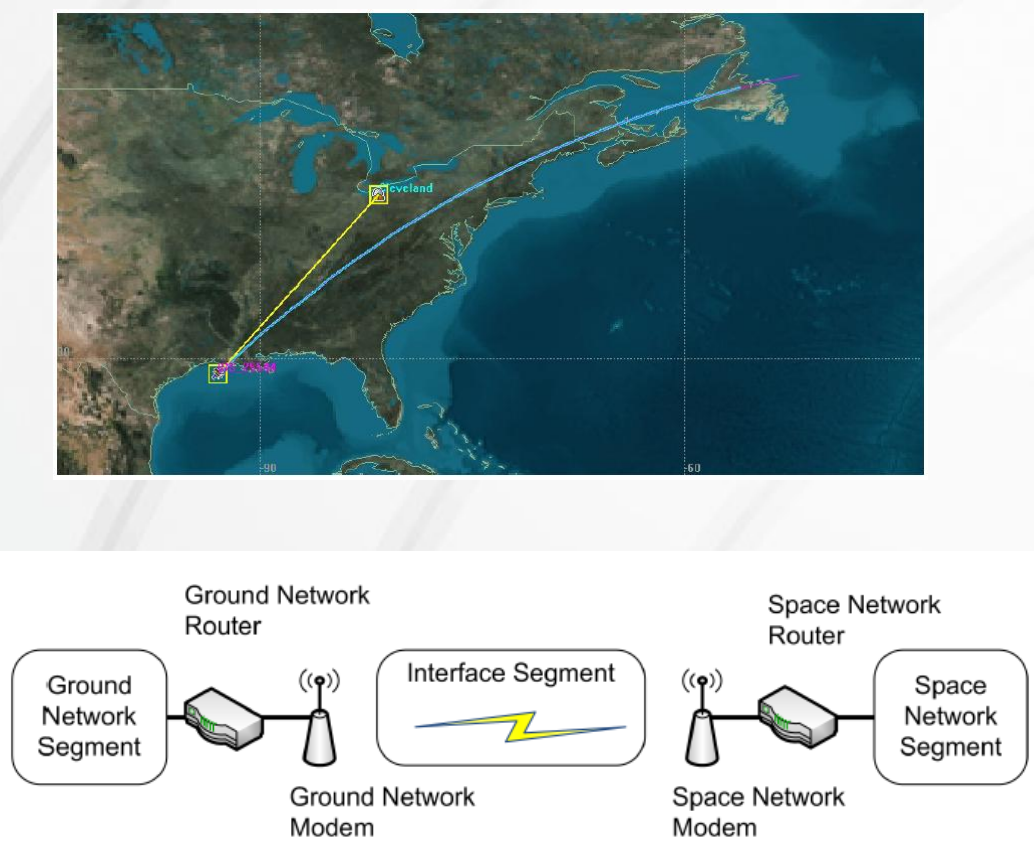

- Bent-Pipe Channel Emulation

- Relay Comm. through Tracking and Data Relay Satellite (TDRS)

- Four Channels:

- Forward to Relay, Forward to Satellite

- Return to Relay, Return to Ground

- Increased latency / path Loss for extended contact time
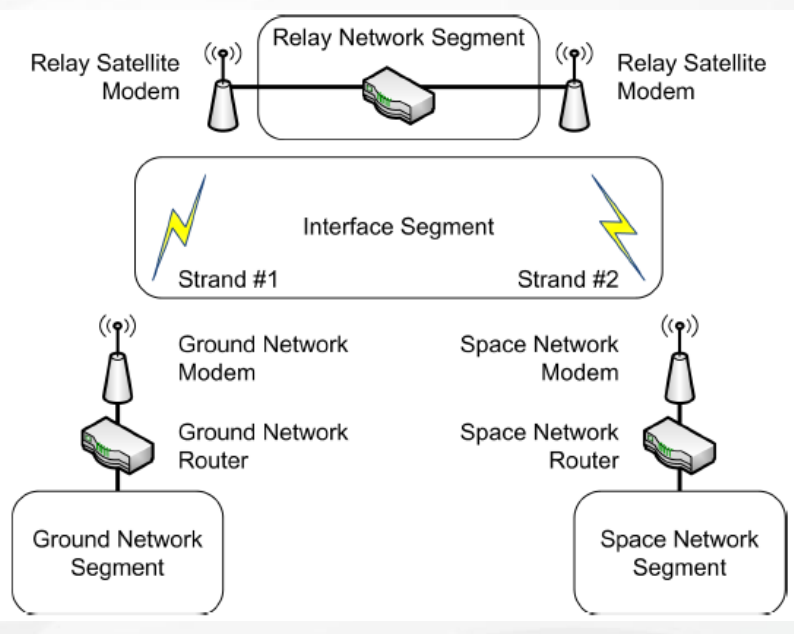


\section{Emulation Hardware}

- Emulation Equipment:

- RT-Logic Channel Simulator

- (2) Satellite Modem

- (2) Routers

- (2) Switches

- User-Equipment (workstations)

- Simulation Equipment:

- STK / EXata Workstations

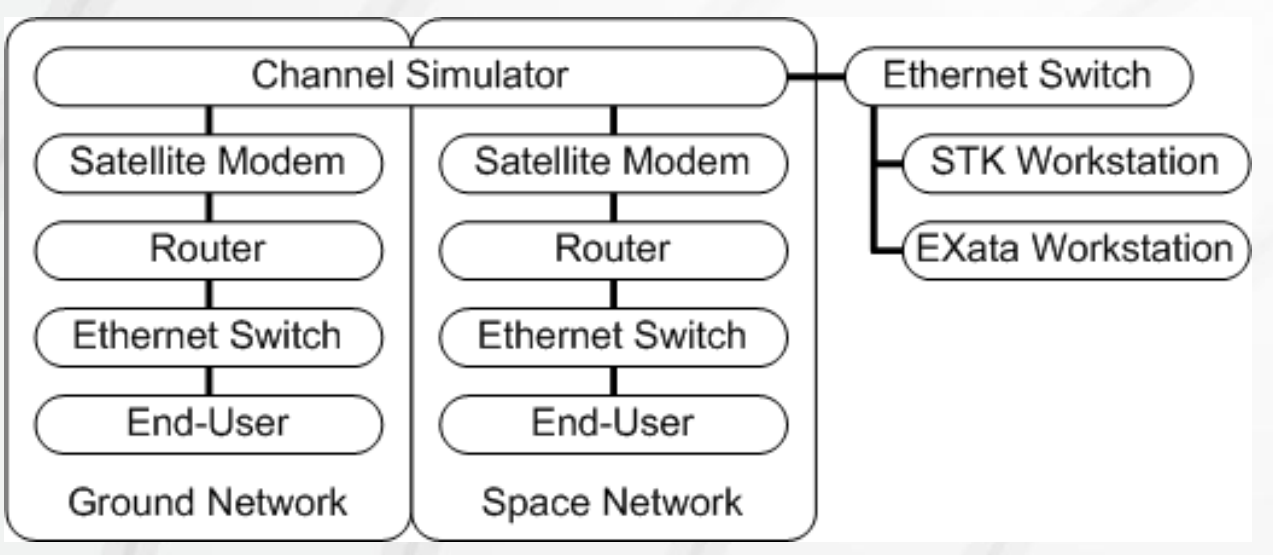

Functional Diagram of Emulation Setup

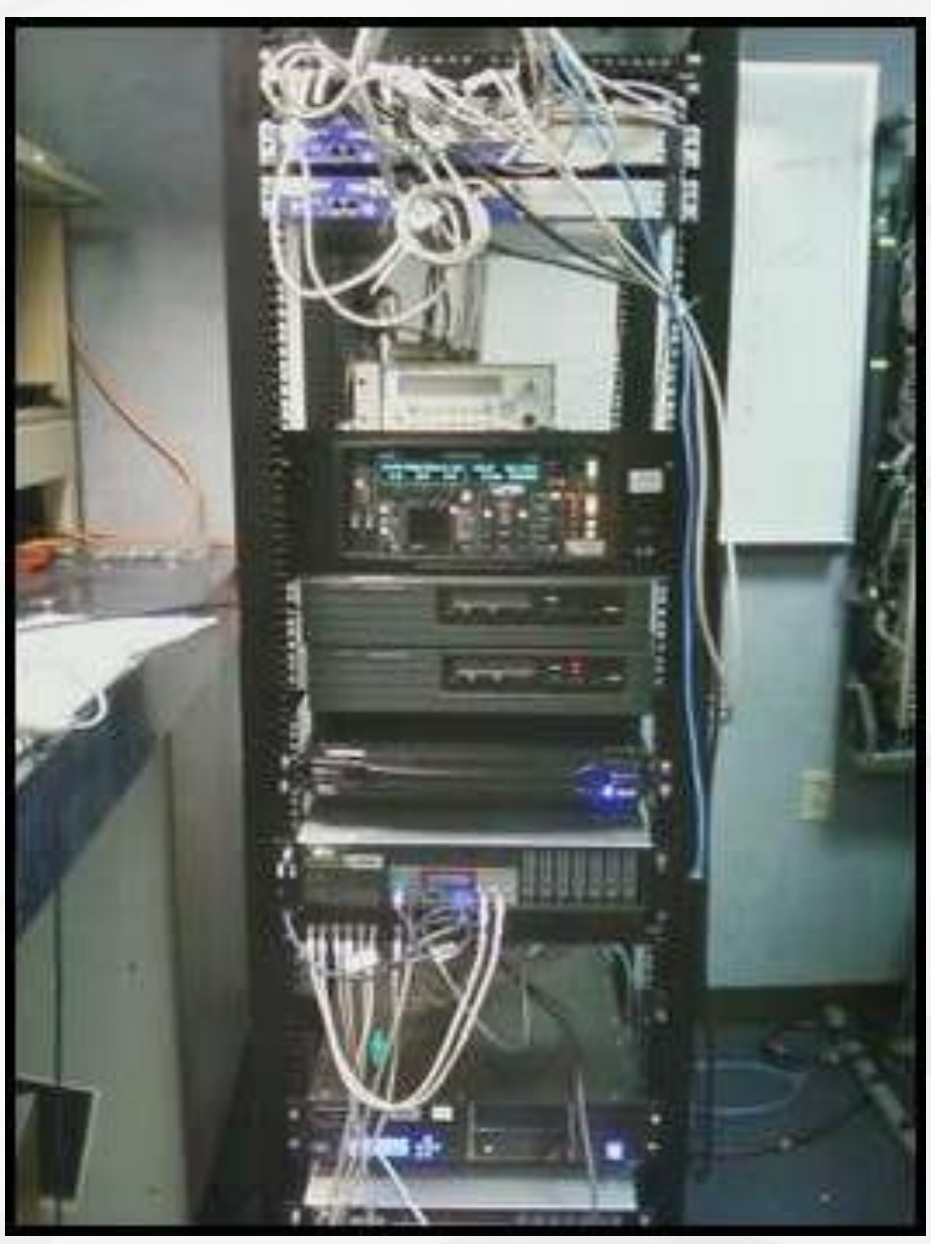

Lab Equipment 


\section{Hardware / Software Integration}

- STK Reports Channel Estimations in Real-time

- Carrier Power at the Receiver (dBW)

- RT-Logic uses this value as the 'Fine Gain' (dB)

- Challenge:

- Develop mapping to compensate for hardware

- Goal:

- Lab modem receive signal strength (RSS) == STK estimated RSS

\begin{tabular}{|c|c|}
\hline \multicolumn{2}{|c|}{ STK Link Budget Calculations } \\
\hline EIRP & $69.04 \mathrm{dBW}$ \\
\hline Free-Space Loss & $-185.82 \mathrm{~dB}$ \\
\hline Atmospheric Loss & $-0.91 \mathrm{~dB}$ \\
\hline Rain Loss & $-13.57 \mathrm{~dB}$ \\
\hline Scintillation Loss & $-2.80 \mathrm{~dB}$ \\
\hline Receiver Gain & $41.04 \mathrm{~dB}$ \\
\hline Carrier Power at Receiver & $-93.02 \mathrm{dBW}$ \\
\hline Carrier Power at Receiver & $-63.02 \mathrm{dBm}$ \\
\hline Receive Signal Strength (w/ 10dB LNA) & $-53.02 \mathrm{dBm}$ \\
\hline
\end{tabular}

\begin{tabular}{|c|c|}
\hline \multicolumn{2}{|c|}{ RT-Logic STK Plugin Offset Calculation } \\
\hline Modem Output Power & $25.0 \mathrm{~dB}$ \\
\hline Pre-processor Gain & $5.0 \mathrm{~dB}$ \\
\hline dBW to dBm Conversion & $30 \mathrm{~dB}$ \\
\hline Cable / Insertion Losses & $1.376 \mathrm{~dB}$ \\
\hline Power Divider Losses & $3.75 \mathrm{~dB}$ \\
\hline LNA Gain & $10.0 \mathrm{~dB}$ \\
\hline Total Offset & $75.126 \mathrm{~dB}$ \\
\hline
\end{tabular}




\section{Hardware / Software Integration}

- Gain offset applied directly to STK estimated Carrier Receive Power

- STK estimated Receive Signal Strength: $-53.02 \mathrm{dBm}$

- Observed Receive Signal Strength: $-53.47 \mathrm{dBm}$

- Observation using Agilent Spectrum Analyzer Channel Power Measurement

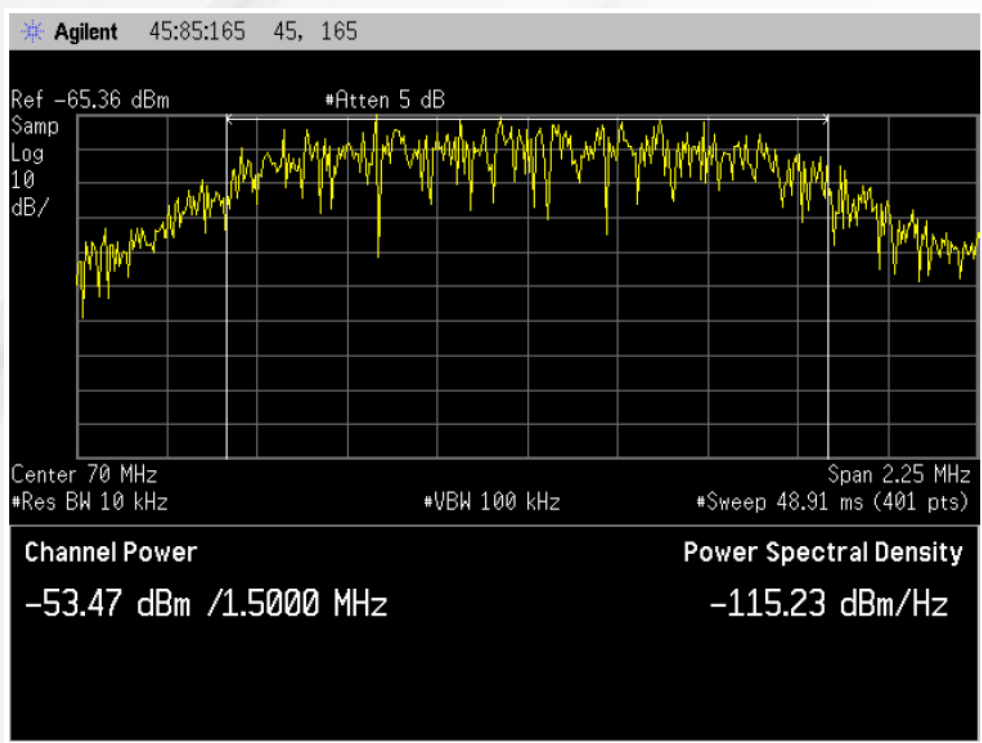

\begin{tabular}{|c|c|}
\hline \multicolumn{2}{|l|}{ RT-Logic Calculations } \\
\hline STK Estimation & $-93.02 \mathrm{~dB}$ \\
\hline Gain Offset & $75.12 \mathrm{~dB}$ \\
\hline Input to Channel Simulator & $-17.9 \mathrm{~dB}$ \\
\hline \multicolumn{2}{|l|}{ Hardware Calculations } \\
\hline Modem Output Power & $-25.0 \mathrm{dBm}$ \\
\hline Cable Losses & $-1.376 \mathrm{~dB}$ \\
\hline Pre-processor Gain & $-5 \mathrm{~dB}$ \\
\hline Power Divider Losses & $-3.75 \mathrm{~dB}$ \\
\hline Channel Simulator Gain & $-17.9 \mathrm{~dB}$ \\
\hline Expected Observed Channel Power & $-53.02 \mathrm{dBm}$ \\
\hline
\end{tabular}




\section{Hardware / Software Integration}

- Other channel simulation characteristics:

- Doppler Shift:

- Apparent shift in frequency due to relative velocity of ground site / satellite

- Noise Floor:

- Power spectral noise of the channel noise
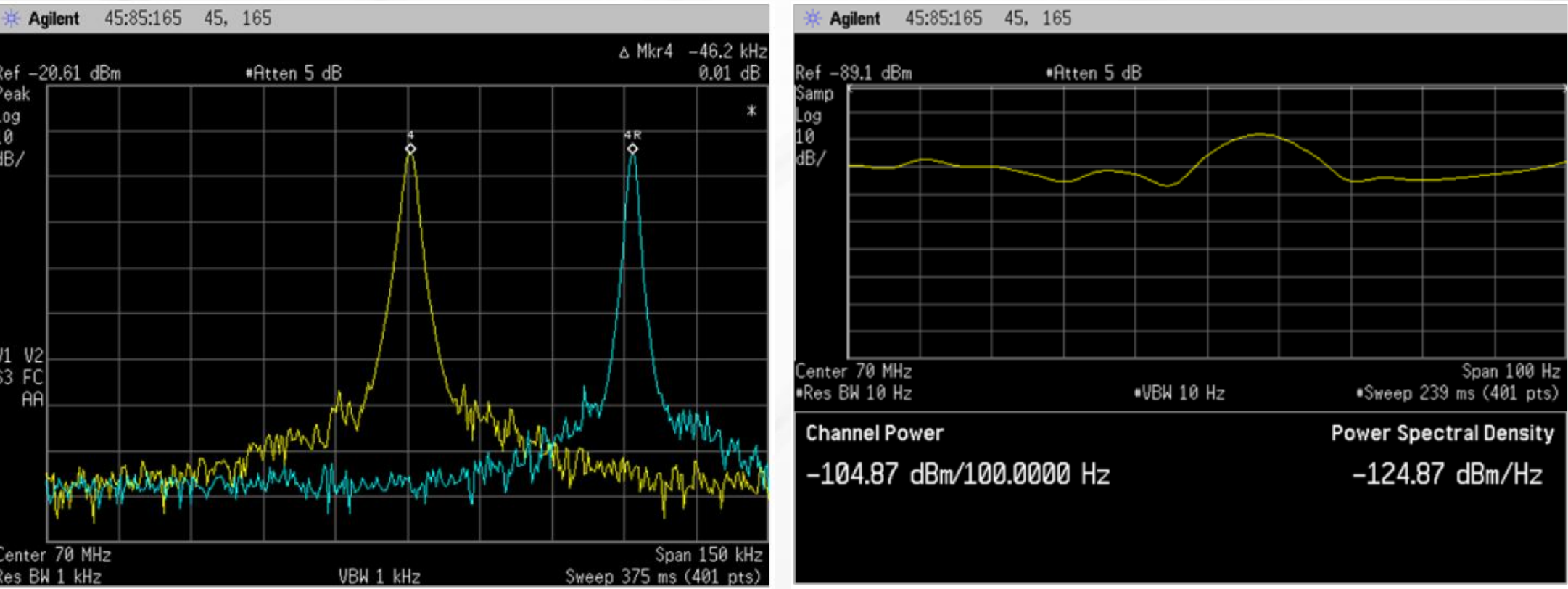


\section{Hardware / Software Integration}

- Channel delay:

- Gathered using ICMP Ping through routers

- Results: Delay follows the estimated STK channel delay

- Additional Delay:

- Modem Delay:

- Channel Coding, Framing

- Router Delay:

- Queuing Delay

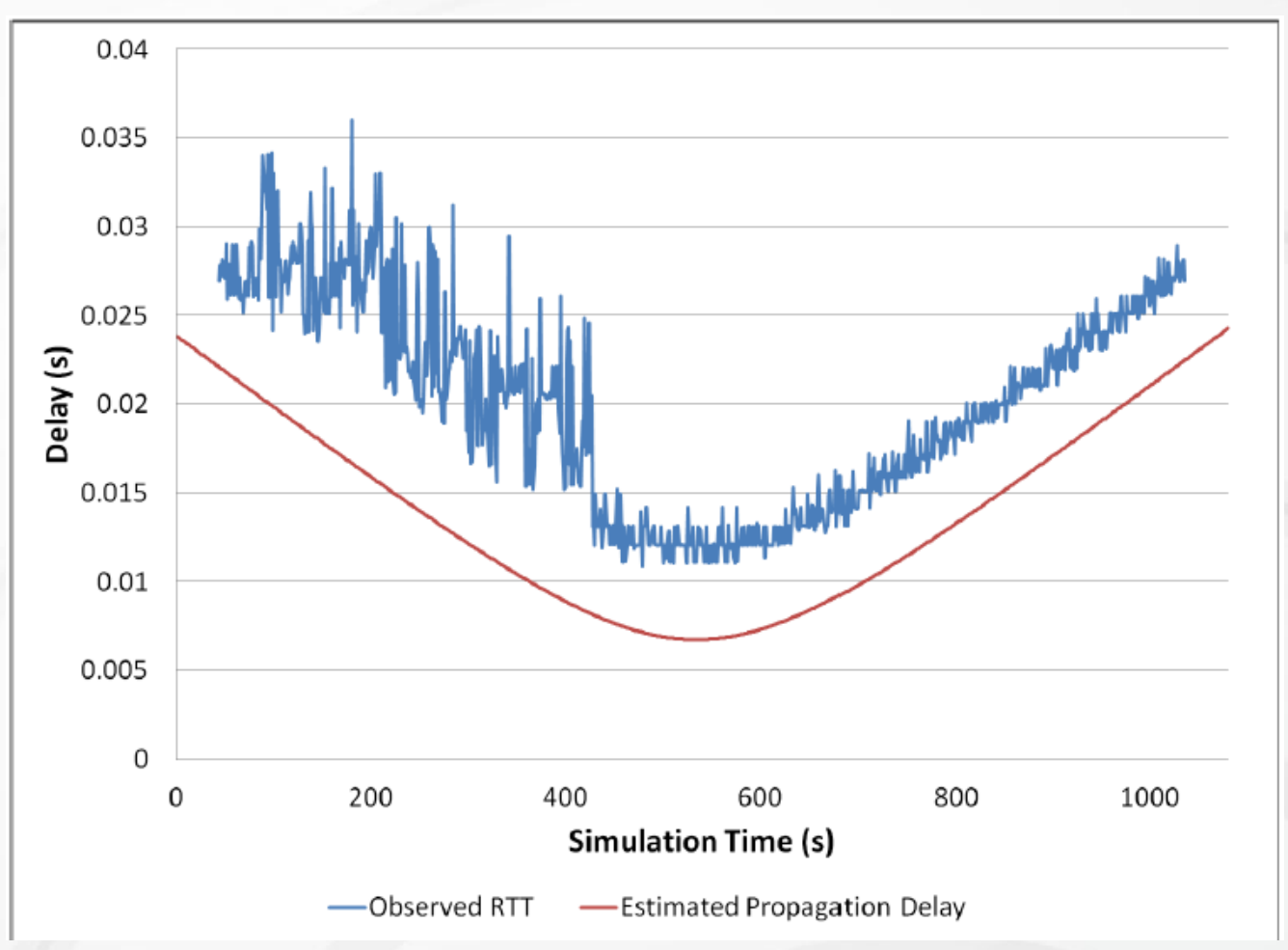




\section{Ongoing Research}

- Integration with network emulation software

- EXata

- Discrete event simulator

- Models network protocols

- Benefits of EXata:

- Software channel modeling

- Can add more channels without additional hardware

- Model ground network

- NASA Integrated Services Network (NISN)

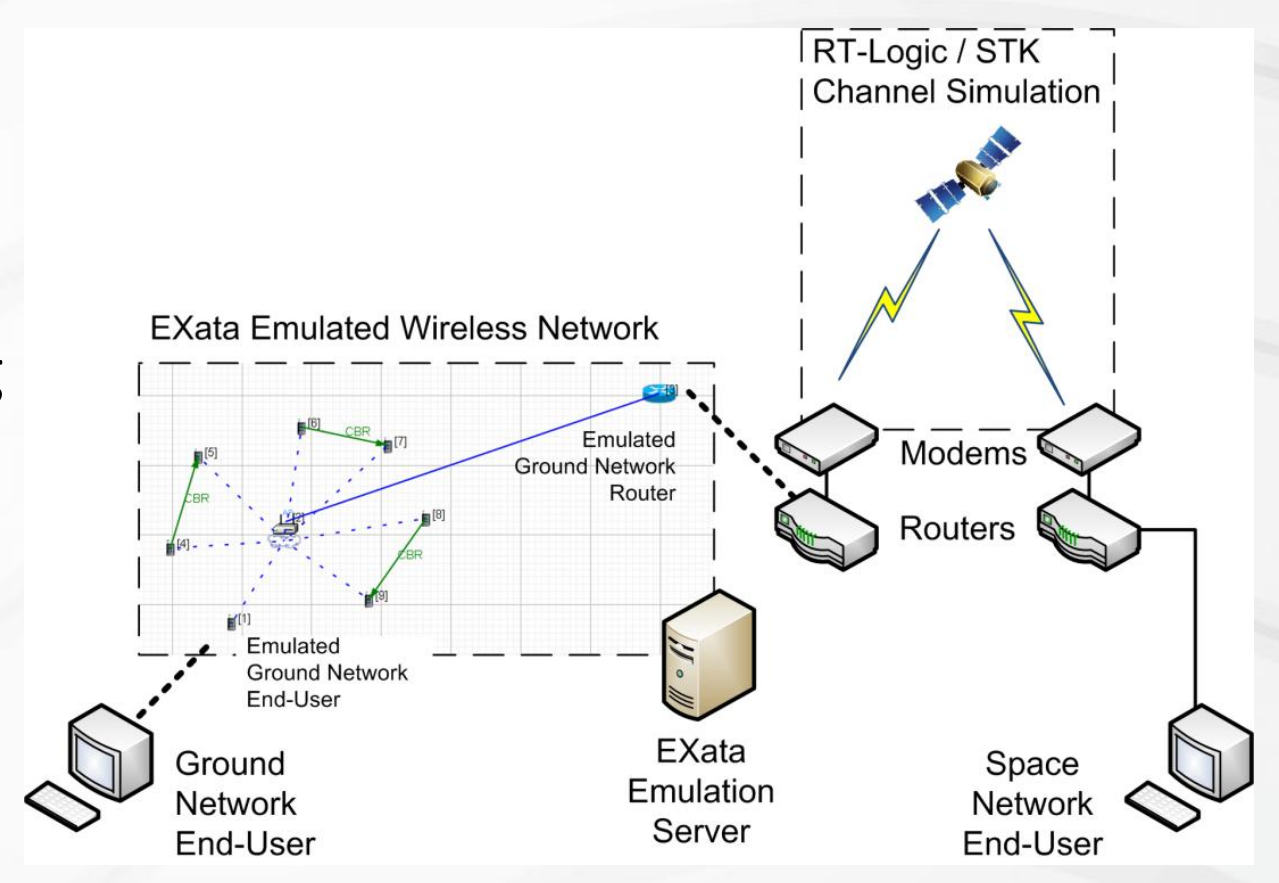




\section{Ongoing Research}

- Current results:

- Excessive delay due to co-simulation: STK/EXata

- Cannot meet real-time simulation requirements

- Coordination between STK/EXata workstations = slow
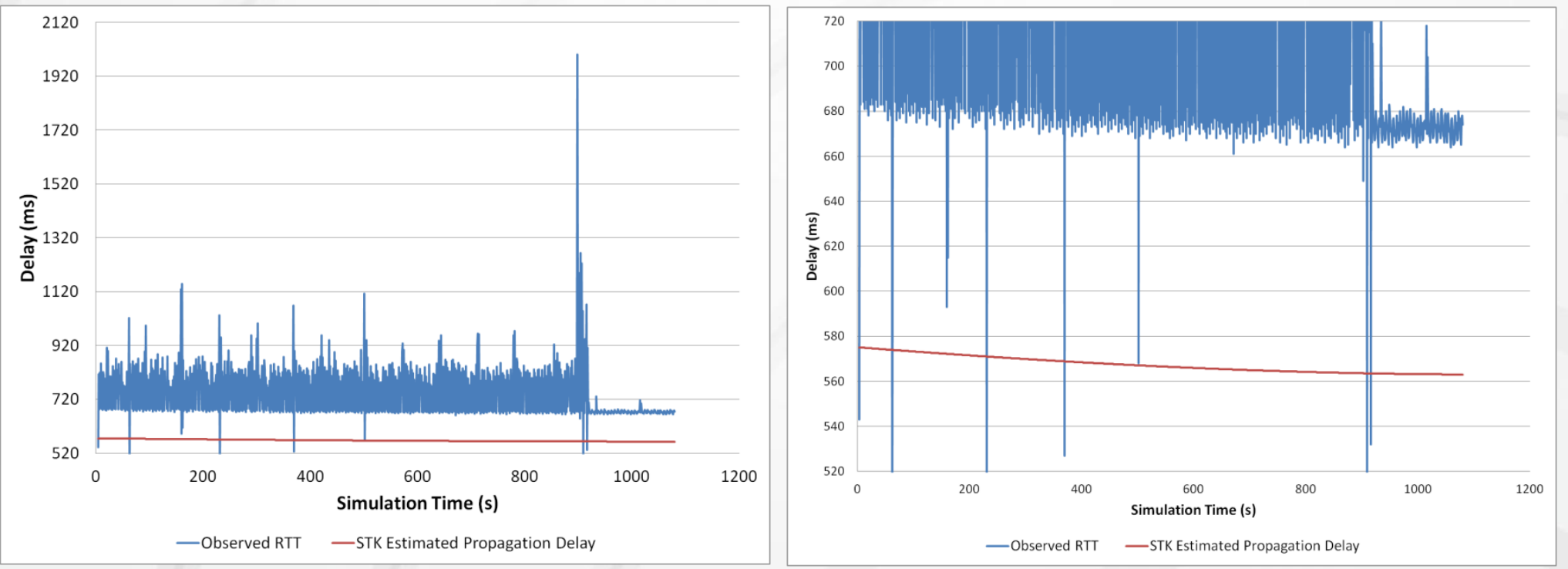


\section{Future Work}

- Improvement to custom EXata / STK interface

- Reduce communication overhead between simulators

- Meet real-time emulation requirement

- Is modeling received signal strength (RSS) sufficient?

- Channel Bit Error Rate (BER) is affected by $E_{b} / N_{0}$

- Noise floor of emulation equipment may not be the same as modeled field hardware. 


\section{Acknowledgements}

This work was completed at NASA Glenn Research Center under the

Space Communication and Navigation (SCaN) Project 
Thank you!

Questions? 